\title{
Global sensitivity analysis of a dynamic model for gene expression in Drosophila embryos
}

Gregory D McCarthy, Robert A Drewell, Jacqueline M Dresch

It is well known that gene regulation is a tightly controlled process in early organismal development. However, the roles of key processes involved in this regulation, such as transcription and translation, are less well understood, and mathematical modeling approaches in this field are still in their infancy. In recent studies, biologists have taken precise measurements of protein and mRNA abundance to determine the relative contributions of key factors involved in regulating protein levels in mammalian cells. We now approach this question from a mathematical modeling perspective. In this study, we use a simple dynamic mathematical model that incorporates terms representing transcription, translation, mRNA and protein decay, and diffusion in an early Drosophila embryo. We perform global sensitivity analyses on this model using various different initial conditions and spatial and temporal outputs. Our results indicate that transcription and translation are often the key parameters to determine protein abundance. This observation is in close agreement with the experimental results from mammalian cells for various initial conditions at particular time points, suggesting that a simple dynamic model can capture the qualitative behavior of a gene. Additionally, we find that parameter sensitivites are temporally dynamic, illustrating the importance of conducting a thorough global sensitivity analysis across multiple time points when analyzing mathematical models of gene regulation. 


\title{
Global sensitivity analysis of a dynamic model for gene expression in Drosophila embryos
}

\author{
Gregory D. McCarthy · Robert A. \\ Drewell · Jacqueline M. Dresch
}

the date of receipt and acceptance should be inserted later

\begin{abstract}
It is well known that gene regulation is a tightly controlled process in early organismal development. However, the roles of key processes involved in this regulation, such as transcription and translation, are less well understood, and mathematical modeling approaches in this field are still in their infancy. In recent studies, biologists have taken precise measurements of protein and mRNA abundance to determine the relative contributions of key factors involved in regulating protein levels in mammalian cells. We now approach this question from a mathematical modeling perspective. In this study, we use a simple dynamic mathematical model that incorporates terms representing transcription, translation, mRNA and protein decay, and diffusion in an early Drosophila embryo. We perform global sensitivity analyses on this model using various different initial conditions and spatial and temporal outputs. Our results indicate that transcription and translation are often the key parameters to determine protein abundance. This observation is in close agreement with the experimental results from mammalian cells for various initial conditions at particular time points, suggesting that a simple dynamic model can capture the qualitative behavior of a gene. Additionally, we find that parameter sensitivites are temporally dynamic, illustrating the importance of conducting a thorough global sensitivity analysis across multiple time points when analyzing mathematical models of gene regulation.
\end{abstract}

Gregory D. McCarthy

Hampshire College, 893 West St, Amherst, MA 01002

E-mail: gm13@hampshire.edu

Robert A. Drewell

Biology Department

Clark University, Worcester, MA 01610

E-mail: rdrewell@clarku.edu

Jacqueline M. Dresch

Department of Mathematics

Amherst College, Amherst, MA 01002

E-mail: jdresch@amherst.edu 


\section{Introduction}

\subsection{Gene Regulation in Embryonic Development}

Embryonic development in animals is very precisely controlled by a network of regulatory proteins $[1,2]$. For any particular protein the exact level of expression at a specific time point can be crucial to the proper development of the organism [3]. Within each cell of the developing embryo, protein abundance is a function of two key molecular events, transcription and translation. Transcription is the process of reading a gene in a DNA template to produce a messenger RNA (mRNA), while translation is the process of reading the mRNA to produce a protein product. A simple, but long-standing question in Biology is the following: Which is contributing more to the variance in protein levels in cells, transcription or translation?

\subsection{Work Analyzing the Importance of Transcription in Mammalian Cells}

Many experimental studies have been conducted in an attempt to understand the roles of transcription and translation in regulating the dynamic nature of mRNA and protein concentrations [4-10]. One such recent detailed study, conducted by the Biggin lab using data from ubiquitously expressed (housekeeping) genes in cultured mammalian cells, aimed to improve existing quantification of protein abundances through statistical analysis of the impact of experimental error [10]. In this study, a two-part regression procedure was used to derive new estimates of protein abundance from the 2011 data set of Schwanhausser et al. [10,6]. Using these new, corrected measurements of protein abundances, along with previous measurements of mRNA and protein degradation rates, they were able to determine the relative importance of transcription, translation, mRNA degradation, and protein degradation (see Figure 1A). This analysis is referred to as the measured protein error strategy. The result of this procedure found that for the 4,212 genes considered, transcription contributed the most to protein abundance $(\sim 38 \%)$, with translation contributing slightly less $(\sim 30 \%)$, followed by mRNA degradation $(\sim 18 \%)$ and protein degradation $(\sim 14 \%)[10]$. This result is important to note, as it differs drastically from Schwanhausser et al.'s original conclusion that translation accounts for the largest contribution $(\sim 55 \%)$ to overall variance in the cellular abundance of proteins $[6,10]$.

\subsection{Existing Mathematical Models of Gene Expression}

Due to the quantitative nature of gene regulation in the embryo, and the advent of new experimental techniques giving rise to massive amounts of mRNA and protein concentration data, various mathematical models have been derived and implemented to help understand the complexity that lies within 
developmental gene regulatory networks. These models range from static models, considering only transcription at a single time point in development in a single cell, to dynamic spatio-temporal models that incorporate transcription, translation, diffusion, and decay rates for a network of genes that regulate one another over a continuous time frame [11-24].

To accurately model protein abundance in a metazoan animal, such as a mouse or fruit fly, one must consider both spatial and temporal dynamics in the developmental system. One such model that we developed uses a discretized reaction-diffusion equation to model concentrations of mRNA and protein in a developing Drosophila embryo across $n$ nuclei [23]. This model not only incorporates terms for mRNA and protein synthesis and decay, but also diffusion of these molecules in the developing embryo. This is particularly important in Drosophila development as the early stages of embryogenesis are marked by 13 mitotic (nuclear) divisions in the absence of cellular divisions, resulting in a multinucleate syncytial embryo (see Figure 1B).

In its simplest form, the model can be written as:

$$
\frac{d y_{a, i}}{d t}=S_{a, i}(Y)+D_{a}\left(y_{a, i+1}-2 y_{a, i}+y_{a, i-1}\right)-\lambda_{a} y_{a, i}
$$

where $a$ represents the specific mRNA or protein that the equation corresponds to, $i$ represents the specific nucleus, $D_{a}$ and $\lambda_{a}$ are the corresponding diffusion and decay rates, and $Y$ represents the entire vector of mRNA and protein concentrations within the system being modeled.

Many similar models have been used to model the expression dynamics of the gap gene system in the developing Drosophila embryo [11,25,26,20,27,21, $28]$. Although these models all rely on an underlying reaction-diffusion framework, they vary greatly in their implementation. Both deterministic $[11,26,20$, $21]$ and stochastic $[25,27,28]$ models have been able to accurately predict the effects of particular perturbations to the network. Stochastic models of hunchback regulation have been used to shed light on the underlying factors that reduce noise and promote stability of the hunchback gradient. These include the number or arrangement of BICOID and KRUPPEL binding sites within the regulatory sequences that control transcription of the hunchback gene as well as protein diffusion $[25,27,28]$. In this study, we focus on the broad impacts of transcription, translation, diffusion, and decay, and do not consider specific transcription factor binding sites within regulatory DNA sequences. Thus, we focus on a deterministic model and do not include any stochasticity.

\subsection{Global Parameter Sensitivity Analysis and HDMR}

To help develop a better understanding of the model parameters, including how their values impact the model output (protein abundance) and how one might interpret that impact with respect to the biological system, parameter sensitivity analysis is needed. Parameter sensitivity analysis refers to a mathematical analysis of the change in model output as a result of variation in 
the input parameter values [29-34]. This analysis can be done locally, at a particular point in parameter space, or globally across the entire parameter space.

Local parameter sensitivity analyses are typically implemented by simply computing or approximating the partial derivative of the objective function at a particular point in parameter space to determine how the function changes locally with respect to small variations of a particular parameter [30,32,35]. The major advantages to adopting a local approach are that it is straightforward, often easy to interpret, and computationally inexpensive. However, a significant limitation of local methods is that when dealing with a large parameter space focusing on a single point in that space may not be representative of much of the overall parameter space. In contrast, global methods allow one to calculate parameter sensitivities while considering the full range of parameter space [34]. Most global methods also have the ability to calculate higher order sensitivities, which capture interactions between parameters. This can be challenging to do with a local method, especially when parameter space is large and many different parameter combinations within that space lead to valid model outputs. Therefore, in this study we focus on a global method for parameter sensitivity analysis for our model of gene expression in the Drosophila embryo.

Higher Dimensional Model Representation (HDMR) is a robust global method for calculating parameter sensitivities [36,32]. This method uses the Monte Carlo integration method to decompose the model output, $f(x)$, often referred to as the objective function, into terms of increasing dimensionality with respect to the parameters $x_{1}, \ldots, x_{N}$ :

$$
f(x)=f_{0}+\sum_{i=1}^{N} f_{i}\left(x_{i}\right)+\sum_{1 \leq i<j \leq N} f_{i j}\left(x_{i}, x_{j}\right)+\cdots+f_{12 \ldots N}\left(x_{1}, x_{2}, \ldots, x_{N}\right)
$$

In the above equation, $f_{0}$ is the main effect, and is approximated by the overall mean of model output over all parameter sets sampled. Each function $f_{i}\left(x_{i}\right)$ is a first order term representing the effect of the parameter $x_{i}$ acting independently on the model output. Each function $f_{i j}\left(x_{i}, x_{j}\right)$ is a second order term representing the effect of the parameters $x_{i}$ and $x_{j}$ on the model output. These terms represent the impact pairwise parameter interactions have on determining the model output.

This approximation is done on a bounded subset of $\mathbb{R}^{N}$, where $N$ is the number of model parameters. The bounded subset represents the parameter space and in each dimension, corresponds to a realistic range for the given parameter. In some applications, the parameter space is known or experimentally determined using empirically determined measurements [36,31]. In other cases, parameter space is chosen based on model assumptions and simulations $[29,37,32-34]$.

One of the main assumptions when using HDMR is that the objective function values are normally distributed [36]. Thus, using the bounded parameter space, one generates a pseudo-random sampling using a Sobol Set [38]. The 
bounded parameter space must be a hypercube in $N$-dimensional space, so it can be normalized to the unit hypercube for sampling. Each set of parameter values is then used as input for a model simulation and the corresponding model output is obtained. Once this sampling has been done, for all parameter sets sampled, HDMR approximates the mean of the output values, $f_{0}$, as well as higher order terms using orthonormal polynomial approximations. Firstand second-order terms are then normalized by the total variance to obtain the main effect of each parameter and the effects of pair-wise parameter interactions, referred to as first- and second-order sensitivity indices respectively. Although this method has the ability to calculate higher-order sensitivities, it has been shown that using only first- and second-order terms is sufficient to approximate the total sensitivity in the system $[39,40,32]$.

In this study, we utilize a global HDMR analysis to investigate the sensitivity of our Drosophila embryo gene expression model to the individual transcription, translation, diffusion, and decay rate parameters and higher-order interactions between these parameters. In addition, we compare our results to those in mammalian cells and other studies that have attempted to model different gene expression systems [10].

\section{Methods}

\subsection{Simplified model and Parameters}

In this study, we use a simplified version of our earlier model [23], which was used to predict both mRNA and protein concentrations along a onedimensional strip of nuclei in a developing Drosophila embryo.

The simplifying assumption applied in the current study is that the gene of interest has spatially uniform transcriptional activity. Thus, the transcription rate is held constant. This allows us to utilize simple numerical solvers such as Euler's method or Runga-Kutta methods, and to measure the relative importance of transcription to the model output using a single parameter, $\sigma$. Note that diffusion is discretized with respect to space and zero flux boundary conditions are used [23]. For $2 \leq i \leq n-1$, where $n$ corresponds to the number of nuclei being modeled, the model equations are thus as follows: 
Table 1 This table describes all model parameters and the ranges used during the sensitivity analysis

\begin{tabular}{lll}
\hline Parameter & Definition & Range \\
\hline$\sigma$ & transcription rate & {$[0.012,2.0]$} \\
$d$ & mRNA diffusion rate & {$[0.0,1.5]$} \\
$\lambda$ & mRNA decay rate & {$[0.0,0.8]$} \\
$\tau$ & translation rate & {$[0.125,1.0]$} \\
$\delta$ & protein diffusion rate & {$[0.0,1.5]$} \\
$\gamma$ & protein decay rate & {$[0.4,0.9]$} \\
\hline
\end{tabular}

$$
\begin{aligned}
\frac{d y_{1}}{d t} & =\sigma+d\left(y_{2}-y_{1}\right)-\lambda y_{1} \\
\frac{d y_{i}}{d t} & =\sigma+d\left(\left(y_{i+1}-y_{i}\right)+\left(y_{i-1}-y_{i}\right)\right)-\lambda y_{i} \\
\frac{d y_{n}}{d t} & =\sigma+d\left(y_{n-1}-y_{n}\right)-\lambda y_{n} \\
\frac{d y_{n+1}}{d t} & =\tau y_{1}+\delta\left(y_{n+2}-y_{n+1}\right)-\gamma y_{n+1} \\
\frac{d y_{n+i}}{d t} & =\tau y_{i}+\delta\left(\left(y_{n+i+1}-y_{n+i}\right)+\left(y_{n+i-1}-y_{n+i}\right)\right)-\gamma y_{n+i} \\
\frac{d y_{2 n}}{d t} & =\tau y_{n}+\delta\left(y_{2 n-1}-y_{2 n}\right)-\gamma y_{2 n}
\end{aligned}
$$

Here, $y_{j}$ represents mRNA concentrations for $1 \leq j \leq n$ and protein concentrations for $n+1 \leq j \leq 2 n$. A schematic of the biological processes incorporated in the model is shown in Figure 1. The model parameters are defined in Table 1. For all of the analysis and results shown in Table 2 and Figures 2-4, 52 nuclear positions are used at approximately 50\% egg height (ventral-dorsal) from approximately $10 \%$ to $90 \%$ egg length (anterior-posterior) and the numerical solver Runga-Kutta 4 is used to approximate the solutions to the system of Ordinary Differential Equations shown above.

\subsubsection{Initial Conditions}

Five different initial conditions are used in this study. Each initial condition corresponds to a different group of spatially expressed genes present in an early Drosophila embryo. The first three initial conditions all correspond to genes that are ubiquitously expressed at spatially uniform levels, such as Zelda in the Drosophila embryo. The only difference between these three initial conditions are the levels of the initial concentrations of mRNA and protein. The initial protein and mRNA concentrations used are $0, \frac{1}{2}$, and 1 . These initial conditions allow us to compare our calculated parameter sensitivities to the contribution to variance in mammalian housekeeping genes measured by $\mathrm{Li}$ et al [10].

The other two initial conditions used in the study are representative of genes that are known to be extremely important to early development in 


\section{PeerJ Reviewing Manuscript}

\section{A. Reaction}

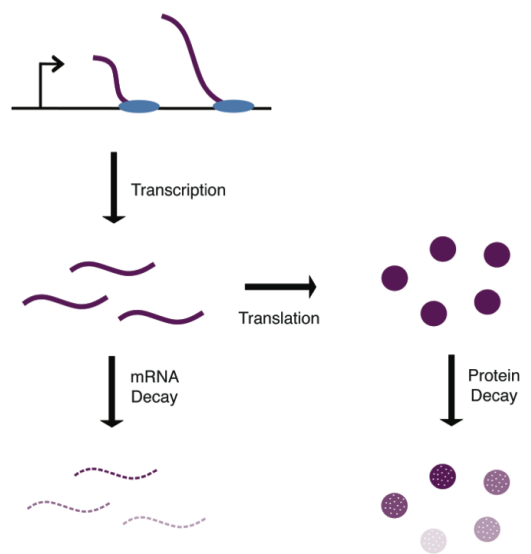

B. Diffusion

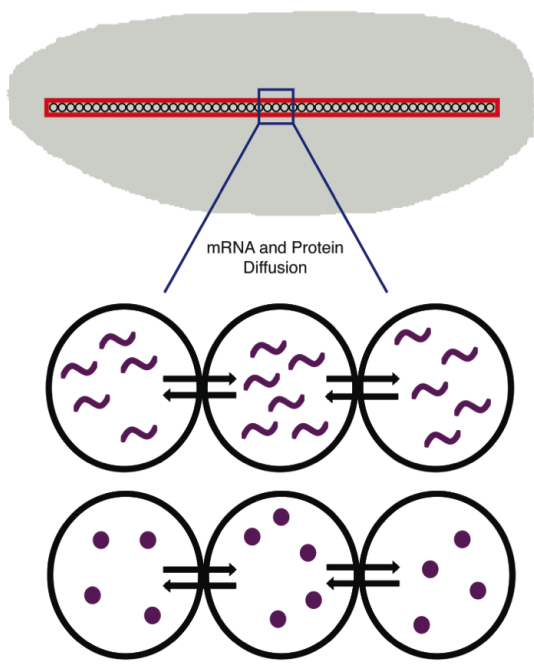

Fig. 1 Schematic of the biological procceses represented in the ODE model. In panel A, the Reaction terms of the model are illustrated. These include the synthesis of new mRNA through transcription, the synthesis of new protein through translation of mRNA, mRNA decay, and protein decay. In panel B, the Diffusion terms of the model are illustrated. These include both mRNA diffusion and protein diffusion to/from neighboring nuclei in an early Drosophila embryo.

Drosopihla embryos, anterior and posteriorly deposited maternal factors, such as Bicoid and Nanos respectively. The initial conditions contain a nonzero mRNA concentration in either the most anterior or posterior nucleus at the initial time point, zero initial mRNA concentrations at all other spatial locations, and zero initial protein concentrations in all nuclei.

\subsection{Exploring Parameter Space}

Before a global parameter sensitivity analysis can be performed, one must first define parameter space. This is done by finding a range for each model parameter that results in 'realistic' model predictions. This will result in a sixdimensional hypercube, as required for the Sobol Set sampling method [38]. For our model, we determine this range for each parameter by exploring sixdimensional parameter space and recording the parameter value combinations resulting in model predictions of reasonable protein concentrations.

The exploration of parameter space is done iteratively on a parameterby-parameter basis. First, we define all protein concentration values $\geq 7$ as protein saturation and $\leq 0.01$ as undetectable protein. The method then works in the following way: 
1. Initial parameter ranges are set to $\sigma, d, \lambda, \delta \in[0,1], \tau \in[0.012,1]$, and $\gamma \in[0.2248,1]$.

Note: Lower bounds were chosen using the values estimated in [23].

2. Choose a single parameter. Test each of the boundaries of the parameter range by holding the parameter of interest constant at the lower (or upper) bound and varying all other parameters within their current ranges. Then, run all simulations for $t \in[0,10]$ with all 5 different initial conditions. If $>5 \%$ of all simulations result in saturated or undetectable protein concentrations, then modify the boundary of the parameter range by increasing or decreasing it by a number between 0.001 and 0.1 .

Note: Lower bounds on parameter ranges were never allowed to go below 0.0 , as negative parameter values would violate the model assumptions.

3. Repeat step 2 for all remaining parameters.

4. When all parameter ranges have been tested, let all 6 parameters vary within their current ranges. If $>5 \%$ of all simulations result in saturated or undetectable protein concentrations, then go back to step 2. If not, then stop.

The realistic parameter ranges we obtained and used for our subsequent sensitivity analyses are listed in Table 1.

\subsection{Objective Function}

To calculate model parameter sensitivies, we utilize a previously developed HDMR MATLAB script [36]. Due to the spatial and temporal dynamics of our model, we perform this analysis using a variety of different objective functions.

We focus our analysis on a twenty minute time window in an early blastoderm embryo. Within this time window, we perform our sensitivity analysis at eleven distinct, evenly distributed time points. This removes any bias in the time point chosen and allows us to look at the behavior of parameter sensitivities over time.

One should note that a parameter's first-order sensitivity is calculated by approximating the variance with respect to that parameter divided by the total variance of the objective function. Thus, at $t=0$, this results in a ratio of $\frac{0}{0}$ since all parameter sets will result in an output equal to the initial condition. Thus, we define the first-order sensitivity of each parameter to be zero when the model output used is from the time point $t=0$.

Spatially, the model aproximates mRNA and protein concentrations at 52 nuclei across the anterio-posterior axis of the embryo. When performing our sensitivity analysis, we focus on protein concentrations only and consider three different spatial locations: a nucleus in the anterior of the embryo, in the posterior of the embryo, or in the center of the embryo. We also perform sensitivity analyses using the mean protein concentration over all 52 nuclei.

To avoid any inherent bias in our results, we compute parameter sensitivities using each of the five initial conditions and each combination of spatial and 


\section{PeerJ Reviewing Manuscript}

Table 2 This table contains the first-order sensitivities at the middle nucleus at $t=4$ minutes for a ubiquitous gene with initial concentrations of 1.0 .

\begin{tabular}{lll}
\hline & First-Order Sensitivity & $\begin{array}{l}\text { \% Contribution to Variance } \\
\text { (Li et al 2014) }\end{array}$ \\
\hline 1. Transcription & 0.32 & 0.38 \\
2. mRNA Diffusion & 0.00 & $\mathrm{~N} / \mathrm{A}$ \\
3. mRNA Decay & 0.17 & 0.18 \\
4. Translation & 0.32 & 0.30 \\
5. Protein Diffusion & 0.00 & $\mathrm{~N} / \mathrm{A}$ \\
6. Protein Decay & 0.05 & 0.14 \\
\hline
\end{tabular}

temporal protein concentrations. Thus, the results shown are represenatitive of 120 runs of the HDMR algorithm.

\section{Comparing Model Sensitivities to Experimental Data}

We begin our analysis by comparing the sensitivities for all six model parameters to previously defined contributions of these processes [10]. For all inititial conditions, we identify a time point in development such that our model parameter sensitivities are qualitatively similarly to the calculated contribution to protein abundance from Li et al. [10] (Figure 2). However, we do observe minimal quantitative differences. When modeling a ubiquitous gene with initial concentrations of one and calculating sensitivities using model output at $t=4$, we find that the first-order sensitivities differ by a maximum of \pm 0.09 , with a Pearson correlation coefficient of $>0.96$ between the first-order sensitivities and the calculated contribution to variance in protein levels from Li et al. [10]. When modeling an anterior maternally deposited gene and calculating sensitivities using model output at $t=2$, we find that the first-order sensitivities differ by a maximum of \pm 0.15 , with a Pearson correlation coefficient of $>0.89$ between the first-order sensitivities and the calculated contribution to variance in protein levels from Li et al. [10]. These small observed differences could be due to a number of biological reasons, including noise in the experimental data, species to species variation, or variation in the time point in development in which different genes are expressed. The sensitivities in Figure 2 are similar to those obtained for all other runs of the model tested (i.e. posterior maternally deposited and ubiquitous genes with other initial concentration values).

To determine whether or not parameter interactions play a significant role in the model's behavior, in addition to computing first-order sensitivites, we also consider second-order and total sensitivities (Figure 2). Here, note that second-order sensitivities account for a very small portion $(<12 \%)$ of the total sensitivity for each parameter. Including second-order sensitivities allows us to account for $99 \%$ of total model sensitivity. Therefore, as has been done in past implementations of HDMR, the sum of first- and second-order sensitivities is shown as an approximation to the total sensitivity of each parameter $[39,40$, 32]. However, one should note that first-order sensitivities alone account for 
over $85 \%$ of total sensitivity. This indicates that a great deal of information regarding the contribution of each parameter to the overall behavior of the gene expression system can be gleaned from the first-order sensitivities, which are in strong agreement with the experimental data from Li et al. [10] (Figure 2).

\section{Dynamic Parameter Sensitivities}

Due to the dynamic nature of Drosophila development, we also analyze parameter sensitivities at multiple different time points. Figure 3 contains firstorder sensitivities corresponding to model simulations with the same initial conditions as those used in the analysis of a ubiquitously expressed anteriorlydeposited mRNA in Figure 2, but calculated using model output at a later time point $(t=10$ in both cases). One should note that qualitatively, the parameter sensitivities have changed drastically. The sensitivity of $\lambda$, the parameter representing mRNA decay, has increased significantly in both cases (from 0.17 to 0.31 for a ubiquitous gene and 0.03 to 0.28 for an anterior deposited gene) while the sensitivities of both $\sigma$ and $\tau$, parameters representing transcription and translation, have decreased. This stark difference from the sensitivities shown in Figure 2 suggests that one should further investigate the dynamic nature of the parameter sensitivites with respect to this model.

To better understand how parameter sensitivities are changing over the twenty minute time interval in which we run the model, we choose eleven different evenly distributed time points $(t=0,2,4,6,8,10,12,14,16,18,20)$. For each set of initial conditions, the HDMR algorithm is implemented using protein concentrations from each of the eleven time points to calculate the parameter sensitivities. The results corresponding to model simulations with the same initial conditions as those used for the analysis in Figures 2-3 are shown in Figure 4 for each model parameter as a function of the time point used for the HDMR analysis.

When considering the dynamics of parameter sensitivities, the general trend observed in all results obtained using nonzero initial conditions is that the model is most sensitive to changes in $\sigma$ and $\tau$, parameters representing transcription and translation, at earlier time points, and becomes more sensitive to $\lambda$, the parameter representing mRNA decay, at later time points (Figure 4).

In Figure 4A, the model begins with nonzero mRNA and protein concentrations across all nuclei. Thus, $\sigma$ (transcription) is important, but the model is more sensitive to $\tau$ (translation) initially as translation is increasing the protein concentrations at a rate proportional to the nonzero mRNA concentration (Figure 4). One should also note that the mRNA concentration is increasing at a rate equal to $\sigma$ (transcription) and decreasing at a rate proportional to the mRNA concentration, causing the model to become more sensitive to $\lambda$ (mRNA decay) and $\sigma$ (transcription) at later time points $(t>5)$ (Figure 4$)$.

In Figure $4 \mathrm{~B}$, we observe a slightly different trend in the parameter sensitivities during early time points due to the fact that initial mRNA concentra- 


\section{PeerJ Reviewing Manuscript}

A.

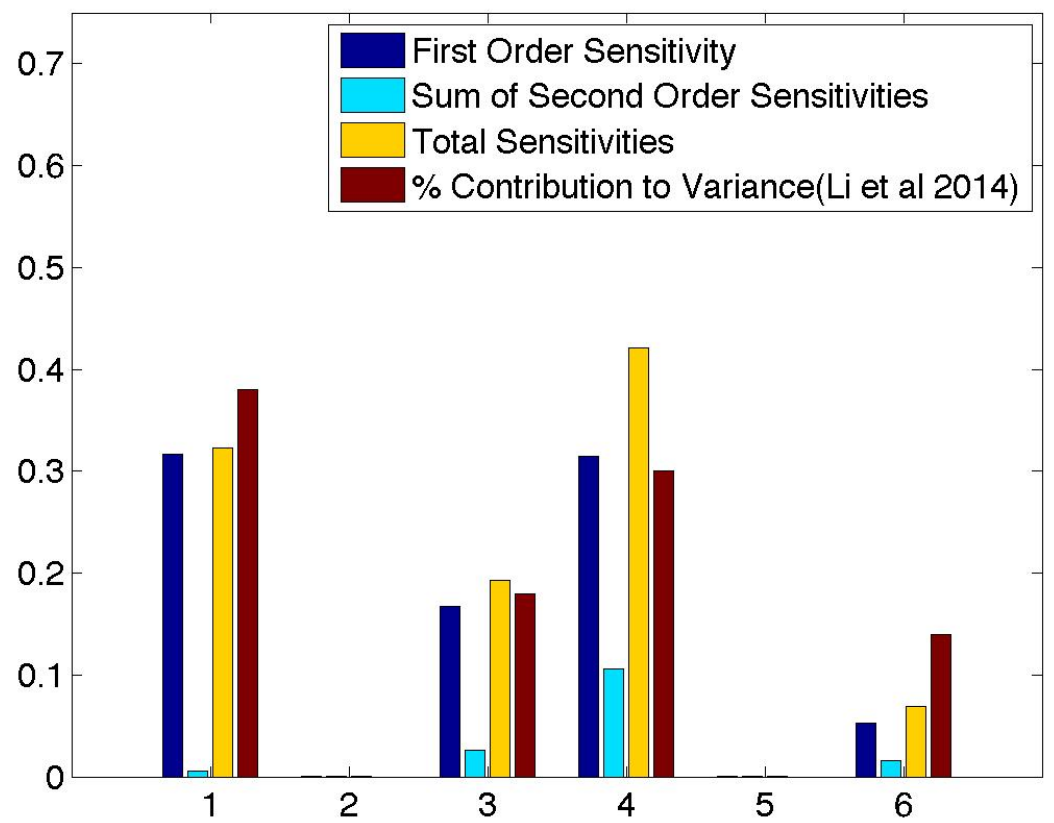

B.

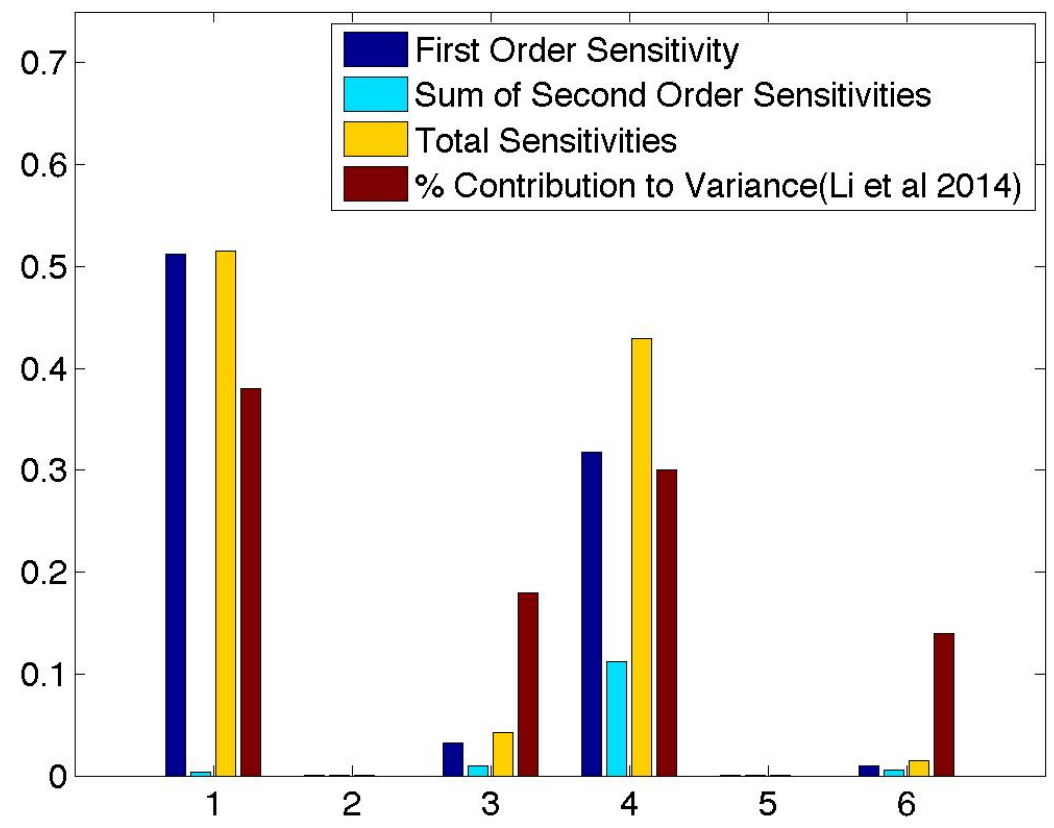

Fig. 2 Qualitative similarities between parameter sensitivities and experimental measurements. A. Ubiquitous gene with initial concentrations of 1.0; First and secondorder sensitivities at the middle nucleus at $t=4$ minutes. B. Anterior maternally deposited gene; First and second-order sensitivities at the middle nucleus at $t=2$ minutes. In both panels, along the x-axis are the parameters corresponding to: 1. Transcription, 2. mRNA Diffusion, 3. mRNA Decay, 4. Translation, 5. Protein Diffusion, and 6. Protein Decay. 
A.

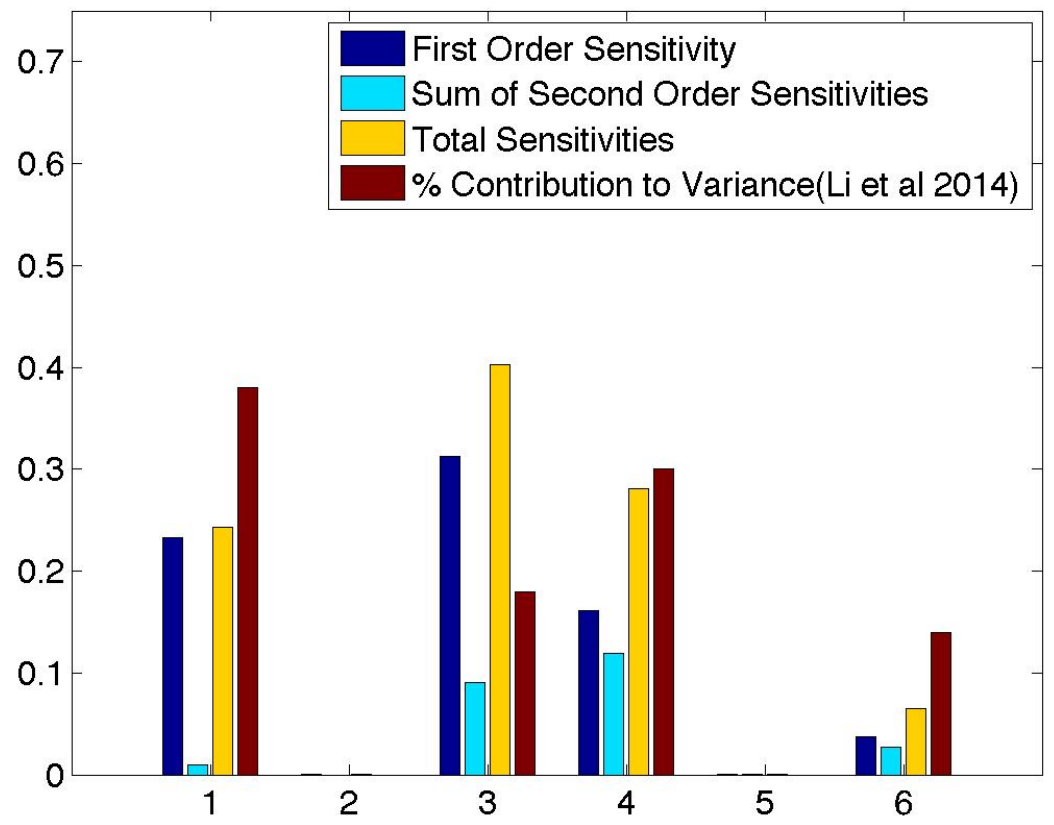

B.

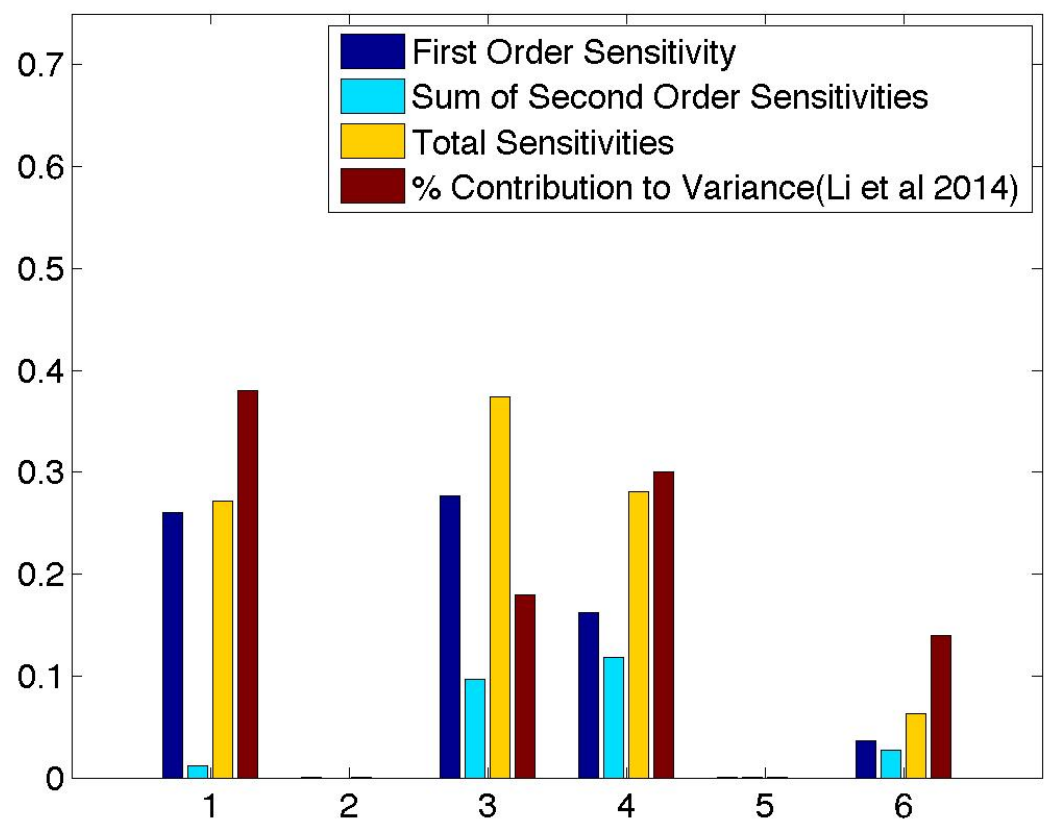

Fig. 3 Comparison of parameter sensitivities to experimental measurements at a later time point. A. Ubiquitous gene with initial concentrations of 1.0; First and secondorder sensitivities at the middle nucleus at $t=10$ minutes. B. Anterior maternally deposited gene; First and second-order sensitivities at the middle nucleus at $t=10$ minutes. In both panels, along the $\mathrm{x}$-axis are the parameters corresponding to: 1 . Transcription, 2. mRNA Diffusion, 3. mRNA Decay, 4. Translation, 5. Protein Diffusion, and 6. Protein Decay. 
tions are zero in all nuclei, except the single most anterior nucleus and protein concentrations are zero in all nuclei. Due to the initial mRNA and protein concentrations of zero in the middle nucleus, and the fact that protein can only be synthesized if the mRNA concentration is greater than zero, the model is more sensitive to $\sigma$ (transcription) than $\tau$ (translation) at early times points. One should note that although the model predictions are quite distinct for the different initial conditions, both predictions show mRNA and protein concentrations in the middle nucleus eventually approaching equilibrium values, and the model exhibits similar relative parameter sensitivities at later time points (Figure 4). Regardless of the qualitative similarities, it is important to note that the dynamic parameter sensitivities are dependent on the initial conditions of the system (Figure 4).

In both cases shown here, the higher model sensitivity with respect to $\sigma$ (transcription) than $\tau$ (translation) at mid to late time points is in agreement with the conclusion of $\mathrm{Li}$ et al. that transcription explains the largest percentage of variance in true protein levels [10]. However, the large contribution from mRNA decay at late time points was not found in the Li et. al. study, as they were unable to consider any dynamic protein levels.

\section{Discussion}

To develop a deeper understanding of the mechanisms involved in regulating the levels of protein concentrations during early development in metazoans, one must consider not only the experimental data that has been carefully collected in the lab, but also the power of mathematical models and the biological interpretation of the parameter values that they use [32,33]. A few very important aspects of modeling that one must consider are how well the model can simulate the reality of the overall system, how well the model agrees with the parts of the system that are already defined biologically, and whether the model parameters can be interpreted in terms of the real biological phenomena that they are assumed to represent. The last of these points is one of the most important, yet remains absent from many modeling studies. In this study, we have used parameter sensitivity analysis to try to unravel the importance of parameter values in a reaction-diffusion model and in doing so to better understand the power of this modeling approach as well as the relative contribution of transcription and translation in regulating protein abundance.

The relative contribution of transcription, translation, and decay rates in overall protein abundance can be aproximated using experimental data [6,10]; however, we ask the question of whether these relative contributions match those found using a mathematical model of gene regulation. We find that the relative parameter sensitivities are in close agreement with the contributions found experimentally $[10]$ for various initial conditions at particular time points (Figure 1). This leads us to believe that even this simple reaction-diffusion model is capturing the correct overall dynamics for a gene with spatially uniform transcriptional activity. 
A.

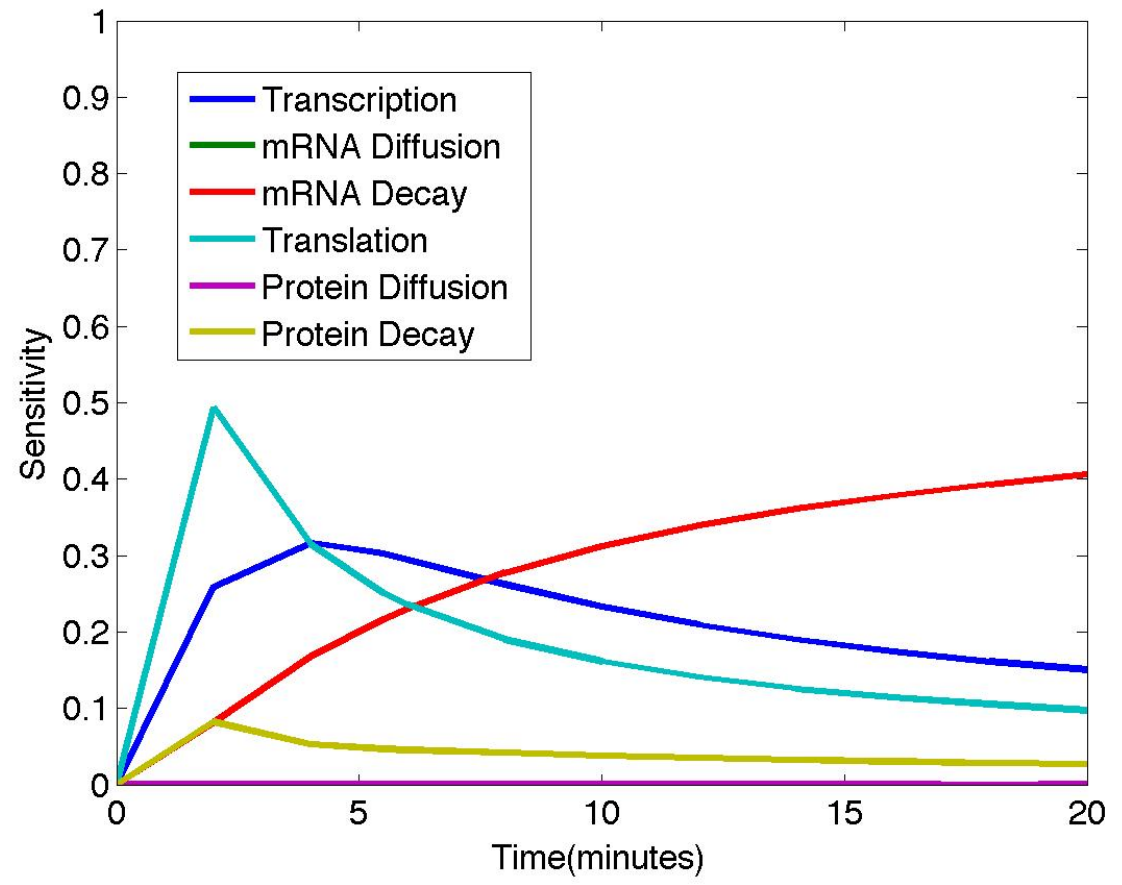

B.

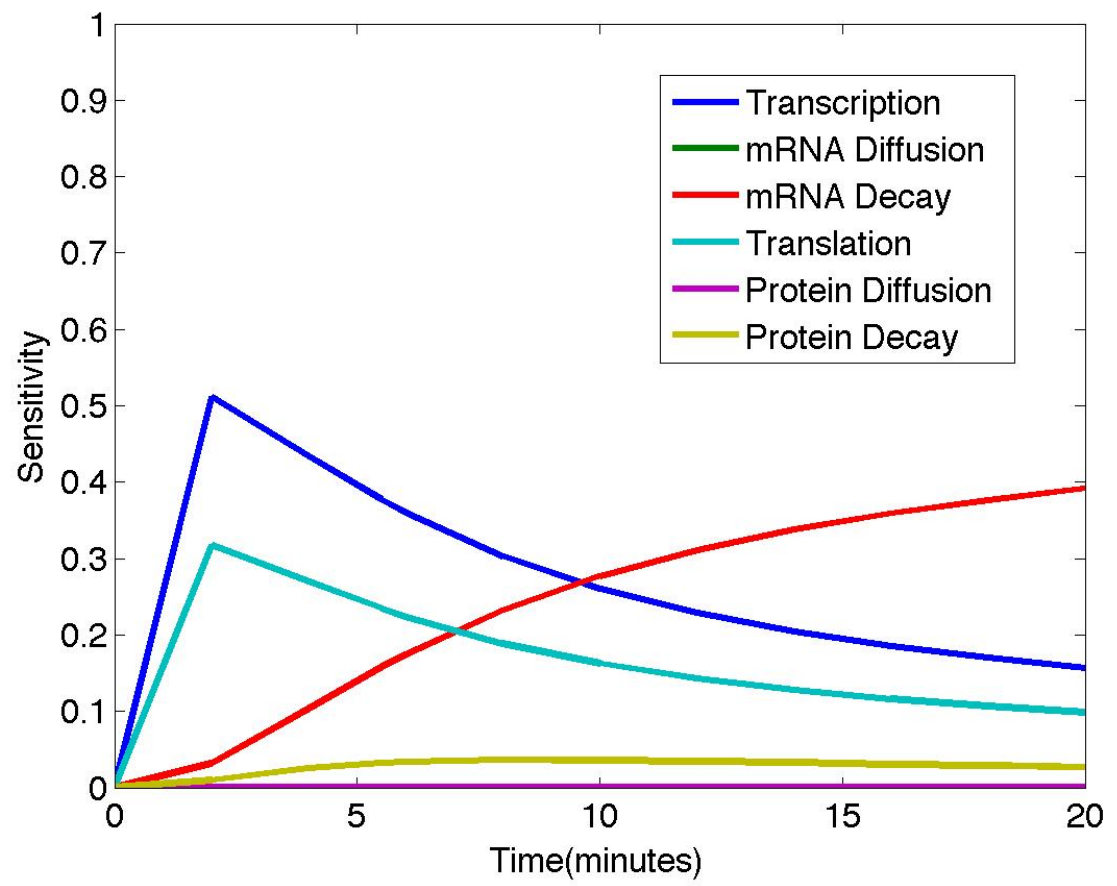

Fig. 4 Temporal dynamics of parameter sensitivities. A. First-order parameter sensitivities at the middle nucleus over time for a ubiquitous gene with initial concentrations of 1.0. B. First-order parameter sensitivities at the middle nucleus over time for an anterior maternally deposited gene 
A number of recent studies have directly addressed the dynamics of gene expression in the model system of the developing Drosophila embryo through quantitative imaging approaches. In Drosophila, the transcriptional activation of the hunchback gene by the BICOID protein in the anterior half of the pre-cellular embryo is itself relatively static from nuclear cycle 10 until midnuclear cycle 14 (approximately 70 minutes) in regions where there is a high concentration of BICOID [41,42]. However, at the posterior limit of hunchback expression, where the BICOID concentration is lower, there is stochastic on/off transcription, suggesting a threshold level of BICOID is required to initiate transcriptional activation. In contrast, the regulation of even-skipped transcription from the stripe 2 enhancer is known to be very dynamic [43]. Although initiated in a broad expression domain in nuclear cycle 11 and 12, transcription becomes increasingly refined in nuclear cycle 13 and 14 to produce a single mature stripe only 2 or 3 nuclei wide. Live imaging studies recently confirmed the dynamic nature of expression directed by the stripe 2 enhancer and demonstrated that the mature stripe is also surprisingly transient (as transcription is lost within 30 minutes, by the end of nuclear cycle 14), with individual nuclei exhibiting discontinuous bursts of transcription [44]. These results emphasize the need to carefully consider the importance of the dynamic spatial and temporal characteristics of gene expression in the networks that regulate embryonic patterning.

Due to the dynamic nature of the Drosophila embryo system, and corresponding mathematical model, we investigated the dynamic nature of parameter sensitivities. These results lead to a very important modeling conclusion and a further biological question. First, one should be cautious when computing parameter sensitivites for a model that is dynamic and a system that is not necessarily in equilibrium, as many protein concentrations are exremely dynamic during early development in an organism. Looking at parameter sensitivities at a single time point can lead to a conclusion that holds for that single time point alone, and the overall behavior of the model may be lost in interpreting these sensitivities individually. Here, we have illustrated the importance of conducting a thorough sensitivity analysis across multiple time points. Second, one must remember the underlying biological question: Which is more important, transcription or translation? In light of the result that relative parameter sensitivites are dynamic, one should reconsider whether this question has a single answer. An interesting question that this study has raised is whether there is a trade-off throughout the early development of the organism. With more experimental data, taken over the course of development, one could ask the detailed question in future studies: Is transcription always contributing more to the variance in protein levels or are there certain points in development where the relative contributions shift? 


\section{Supplemental Information}

The MATLAB source code for the model and numerical solver (Runga-Kutta 4) is contained in file S1. This was used in combination with the HDMR MATLAB code developed by Tilo Ziehn and the MATLAB function sobolset.m, which are both publically available [36]. Additional results for the two other spatial locations used for the sensitivity analysis are shown in figures S1-S6.

\section{References}

1. Davidson, E.: Emerging properties of animal gene regulatory networks. Nature 468(7326), 911-920 (2010)

2. Peter, I., Davidson, E.: Evolution of gene regulatory networks controlling body plan development. Cell 144(6), 970-985 (2011)

3. Davidson, E., Levine, M.: Properties of developmental gene regulatory networks. PNAS 105(51), 20063-20066 (2008)

4. Maier, T., Guell, M., Serrano, L.: Correlation of mrna and protein in complex biological samples. FEBS Letters 583, 3966-3973 (2009)

5. Vogel, C., de Sousa Abreu, R., Ko, D., Le, S., Shapiro, B., Burns, S., Sandhu, D., Boutz, D., Marcotte, E., Penalva, L.: Sequence signatures and mrna concentration can explain two-thirds of protein abundance variation in a human cell line. Molecular Systems Biology 6, $400(2010)$

6. Schwanhausser, B., Busse, D., Li, N., Dittmar, G., Schuchhardt, J., Wolf, J., Chen, W., Selbach, M.: Global quantification of mammalian gene expression control. Nature 473, 337-342 (2011)

7. Beck, M., Schmidt, A., Malmstroem, J., Claassen, M., Ori, A., Szymborska, A., Herzog, F., Rinner, O., Ellenberg, J., Aebersold, R.: The quantitative proteome of a human cell line. Molecular Systems Biology 7, 549 (2011)

8. Bantscheff, M., Lemeer, S., Savitski, M., Kuster, B.: Quantitative mass spectrometry in proteomics: critical review update from 2007 to the present. Analytical and Bioanalytical Chemistry 404, 939-965 (2012)

9. Vogel, C., Marcotte, E.: Insights into the regulation of protein abundance from proteomic and transcriptomic analyses. Nature Reviews Genetics 13, 227-232 (2012)

10. Li, J., Bickel, J., Biggin, M.: System wide analyses have underestimated protein abundances and the importance of transcription in mammals. PeerJ 2:e270; DOI $\mathbf{1 0 . 7 7 1 7 / p e e r j . 2 7 0}(2014)$

11. Jaeger, J., Surkova, S., Blagov, M., Janssens, H., Kosman, D., Kozlov, K., Manu, Myasnikova, E., Vanario-Alonso, C., Samsonova, M., Sharp, D., Reinitz, J.: Dynamic control of positional information in the early Drosophila embryo. Nature 430, 368-371 (2004)

12. Santillan, M., Mackey, M.: Influence of catabolite repression and inducer exclusion on the bistable behavior of the lac operon. Biophysical Journal 86, 1282-1292 (2004)

13. Bintu, L., Buchler, N., Garcia, H., Gerland, U., Hwa, T., Kondev, J., Kuhlman, T., Phillips, R.: Transcriptional regulation by the numbers: applications. Current Opinion in Genetics and Development 15, 125-135 (2005)

14. Janssens, H., Hou, S., Jaeger, J., Kim, A., Myasnikova, E., Sharp, D., Reinitz, J.: Quantitative and predictive model of transcriptional control of the Drosophila melanogaster even skipped gene. Nature Genetics 38, 1159-1165 (2006)

15. Zinzen, R., Senger, K., Levine, M., Papatsenko, D.: Computational models for neurogenic gene expression in the Drosophila embryo. Current Biology 16, 1358-1365 (2006)

16. Segal, E., Raveh-Sadka, T., Schroeder, M., Unnerstall, U., Gaul, U.: Predicting expression patterns from regulatory sequence in drosophila segmentation. Nature 451, $535-540$ (2008)

17. Gertz, J., Siggia, E., Cohen, B.: Analysis of combinatorial cis-regulation in synthetic and genomic promoters. Nature 457, 215-218 (2009) 
18. Fakhouri, W., Ay, A., Sayal, R., Dresch, J., Dayringer, E., Arnosti, D.: Deciphering a transcriptional regulatory code: modeling short-range repression in the Drosophila embryo. Molecular Systems Biology 6, 341 (2010)

19. He, X., Samee, M., Blatti, C., Sinha, S.: Thermodynamics-based models of transcriptional regulation by enhancers: the roles of synergistic activation, cooperative binding and short-range repression. PLoS Computational Biology 6(9), 1000935 (2010)

20. Bieler, J., Pozzorini, C., Naef, F.: Whole-embryo modeling of early segmentation in Drosophila identifies robust and fragile expression domains. Biophysical Journal 101(2), 287-296 (2011)

21. Janssens, H., Crombach, A., Wotton, K., Cicin-Sain, D., Surkova, S., Lim, C., Samsonova, M., Akam, M., Jaeger, J.: Lack of tailless leads to an increase in expression variability in drosophila embryos. Dev. Biol. 377(1), 305-317 (2013)

22. Ilsley, G., Fisher, J., Apweiler, R., Depace, A., Luscombe, N.: Cellular resolution models for even skipped regulation in the entire drosophila embryo. eLife 2:e00522. doi: 10.7554/eLife.0052 (2013)

23. Dresch, J., Thompson, M., Arnosti, D., Chiu, C.: Two-layer mathematical modeling of gene expression: Incorporating dna-level information and system dynamics. SIAM J. Appl. Math. 73(2), 804-826 (2013)

24. Samee, M., Sinha, S.: Quantitative modeling of a gene's expression from its intergenic sequence. PLoS Comput Biol. 10(3), 1003467 (2014)

25. Okabe-Oho, Y., Murakami, H., Oho, S., Sasai, M.: Stable, precise, and reproducible patterning of bicoid and hunchback molecules in the early Drosophila embryo. PLoS Comp. Biol. 5(8), 1000486 (2009)

26. Ashyraliyev, M., Siggens, K., Janssens, H., Blom, J., Akam, M., Jaeger, J.: Gene circuit analysis of the terminal gap gene huckebein. PLoS Comp. Biol. 5(10), 1000548 (2009)

27. Holloway, D., Lopes, F., da Fontoura Costa, L., Travencolo, B., Golyandina, N., Usevich, K., Spirov, A.: Gene expression noise in spatial patterning: hunchback promoter structure affects noise amplitude and distribution in Drosopihla segmentation. PLoS Comp. Biol. 7(2), 1001069 (2011)

28. Holloway, D., Spirov, A.: Mid-embryo patterning and precision in Drosophila segmentation: Kruppel dual regulation of hunchback. PLoS One doi: 10.1371/journnal.pone.0118450 (2015)

29. Frey, H., Patil, S.: Identification and review of sensitivity analysis methods. Risk Analysis 22, 553-578 (2002)

30. van Riel, N.: Dynamic modeling and analysis of biochemical networks: mechanism-based models and model-based experiments. Briefings in Bioinformatics 7, 364-374 (2006)

31. Tang, Y., Reed, P., Wagener, T., van Werkhoven, K.: Comparing sensitivity analysis methods to advance lumped watershed model identification and evaluation. Hydrology and Earth System Sciences 3, 3333-3395 (2006)

32. Dresch, J., Liu, X., Arnosti, D., Ay, A.: Thermodynamic modeling of transcription: sensitivity analysis differentiates biological mechanism from mathematical model-induced effects. BMC Systems Biology 4, 142 (2010)

33. Ay, A., Arnosti, D.: Mathematical modeling of gene expression: a guide for the perplexed biologist. Critical Reviews in Biochemistry and Molecular Biology 46(2), 137 151 (2011)

34. Jarrett, A., Liu, Y., Cogan, N., Hussaini, M.: Global sensitivity analysis used to interpret biological experimental results. J. Math. Biol. doi: 10.1007/s00285-014-0818-3 (2014)

35. Reeves, G., Fraser, S.: Biological systems from an engineer's point of view. PLoS Biol. 7, 32-35 (2009)

36. Ziehn, T., Tomlin, A.: A software tool for global sensitivity analysis of complex models. Environmental Modelling and Software 24(7), 775-785 (2009)

37. Gutenkunst, R., Waterfall, J., Casey, F., Brown, K., Myers, C., Sethna, J.: Universally sloppy parameter sensitivities in systems biology models. PLoS Comp. Biol. 3(10), 189 (2007)

38. Sobol, I.: On the distribution of points in a cube and the approximate evaluation of integrals. USSR Comp. Math. and Math. Physics 7, 86-112 (1976)

39. Li, G., Rosenthal, C., Rabitz, H.: High dimensional model representations. J Phys Chem A 105, 7765-7777 (2001) 
40. Liang, X., Guo, J.: Intercomparison of land-surface parameterization schemes: Sensitivity of surface evergy and water fluxes to model parameters. J Hydrol 279, 182-209 (2003)

41. Garcia, H., Tikhonov, M., Lin, A., Gregor, T.: Quantitative imaging of transcription in living Drosophila embryos links polymerase activity to patterning. Curr. Biol. 23(21), 2140-2145 (2013)

42. Lucas, T., et. al.: Live imaging of bicoid-dependent transcription in Drosophila embryos. Curr. Biol. 23(21), 2135-2139 (2013)

43. Small, S., Blair, A., Levine, M.: Regulation of even-skipped stripe 2 in the Drosophila embryo. EMBO J. 11(11), 4047-4057 (1992)

44. Bothma, J., Garcia, H., Esposito, E., Schlissel, G., Gregor, T., Levine, M.: Dynamic regulation of eve stripe 2 reveals transcriptional bursts in living Drosophila embryos. PNAS 111(29), 10598-10603 (2014) 


\section{1}

Schematic of the biological procceses represented in the ODE model.

In panel A, the Reaction terms of the model are illustrated. These include the synthesis of new mRNA through transcription, the synthesis of new protein through translation of mRNA, mRNA decay, and protein decay. In panel B, the Diffusion terms of the model are illustrated. These include both mRNA diffusion and protein diffusion to/from neighboring nuclei in an early Drosophila embryo.

\section{A. Reaction}
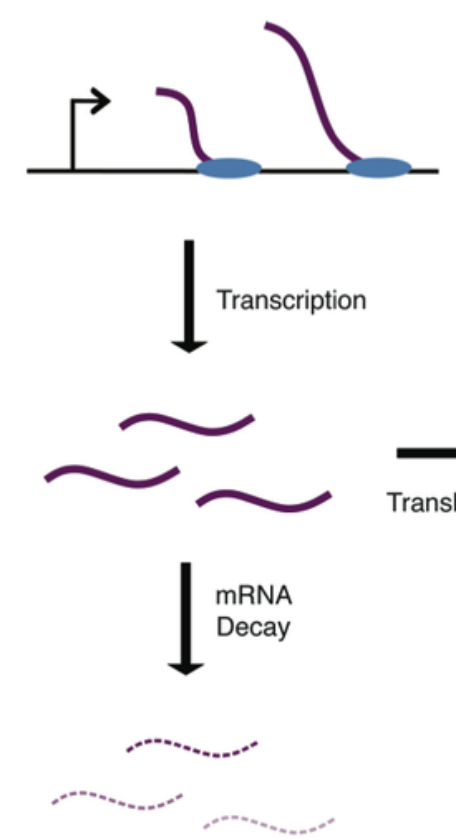

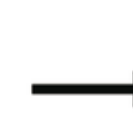

Translation
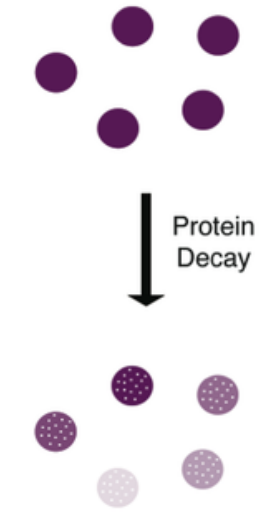

\section{B. Diffusion}

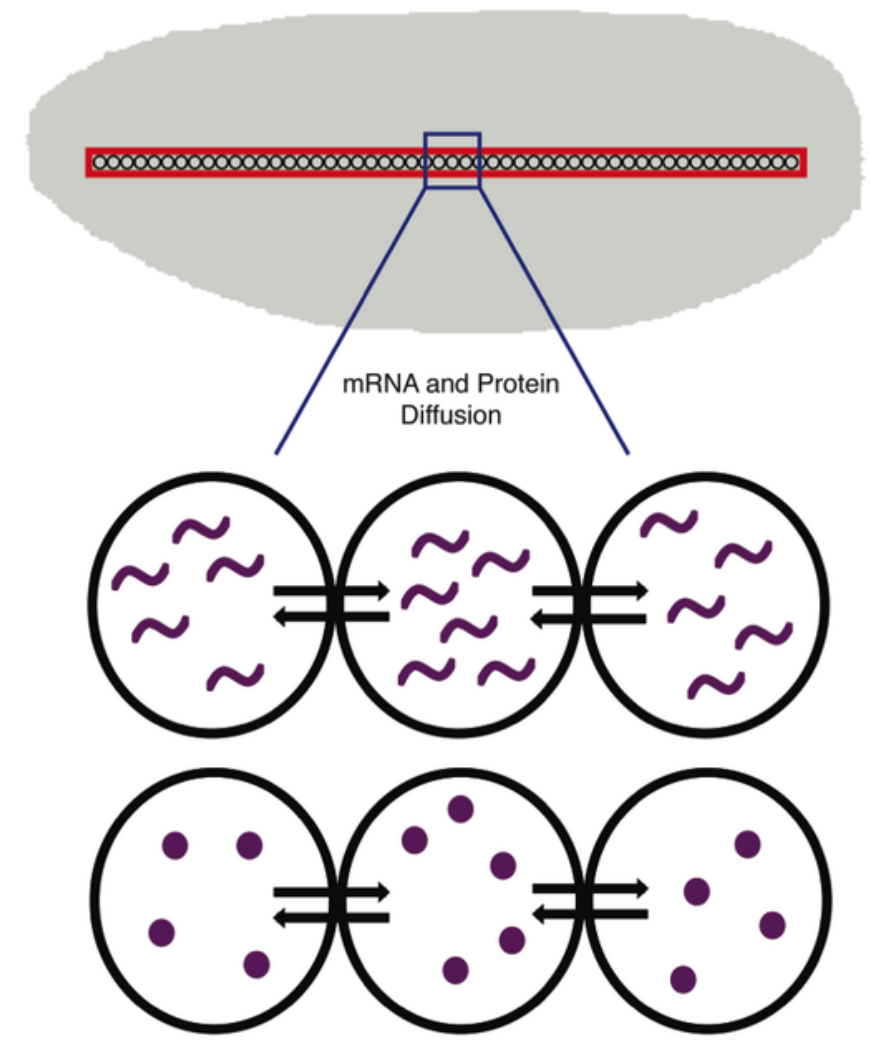


2

Qualitative similarities between parameter sensitivities and experimental measurements.

A. Ubiquitous gene with initial concentrations of 1.0; First and second-order sensitivities at the middle nucleus at $\mathrm{t}=4$ minutes. $\mathrm{B}$. Anterior maternally deposited gene; First and secondorder sensitivities at the middle nucleus at $\mathrm{t}=2$ minutes. In both panels, along the $\mathrm{x}$-axis are the parameters corresponding to: 1. Transcription, 2. mRNA Diffusion, 3. mRNA Decay, 4. Translation, 5. Protein Diffusion, and 6. Protein Decay. 
A.

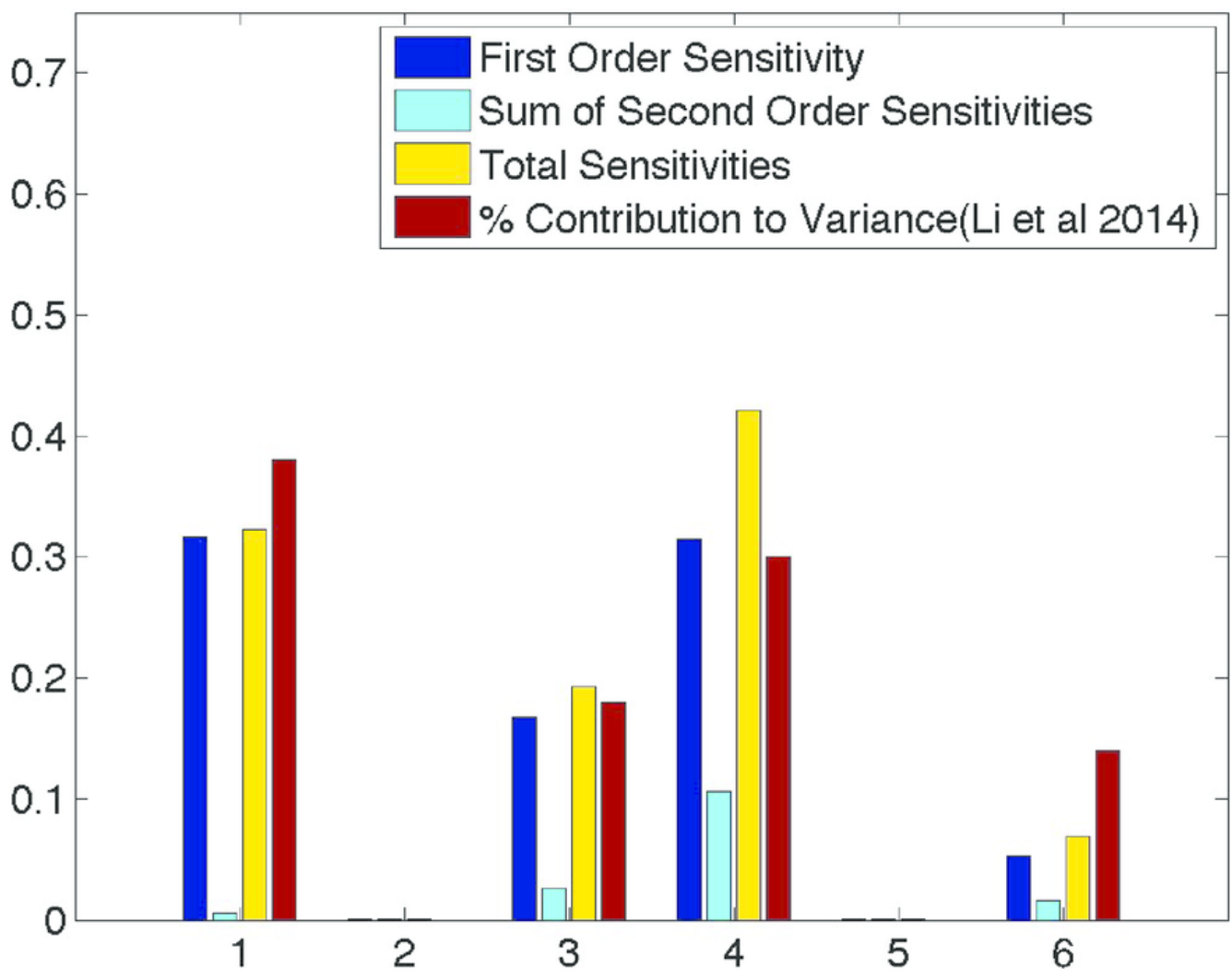

B.

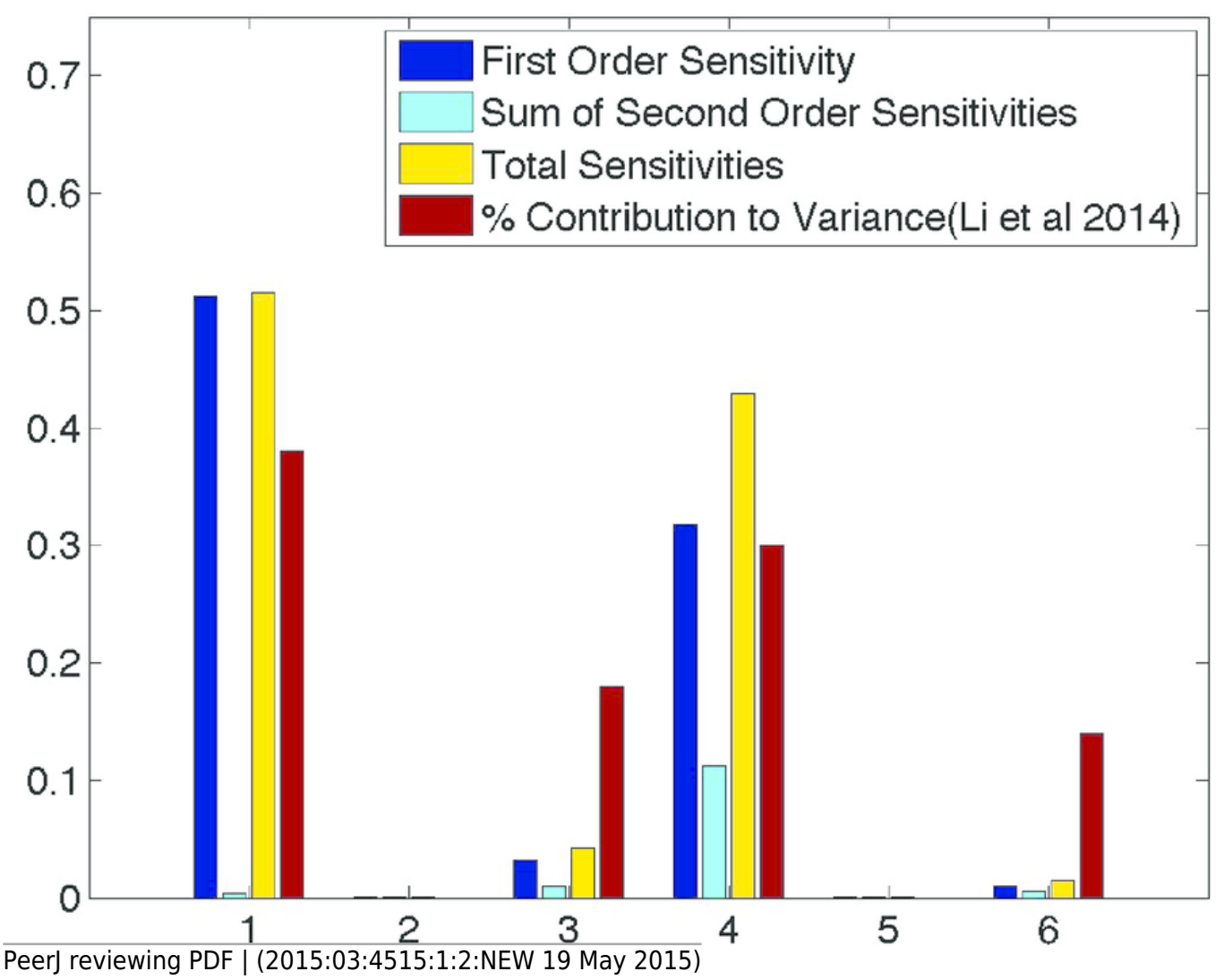




\section{3}

Comparison of parameter sensitivities to experimental measurements at a later time point.

A. Ubiquitous gene with initial concentrations of 1.0; First and second-order sensitivities at the middle nucleus at $\mathrm{t}=10$ minutes. $\mathrm{B}$. Anterior maternally deposited gene; First and second-order sensitivities at the middle nucleus at $t=10$ minutes. In both panels, along the $x$-axis are the parameters corresponding to: 1. Transcription, 2. mRNA Diffusion, 3. mRNA Decay, 4. Translation, 5. Protein Diffusion, and 6. Protein Decay. 
A.

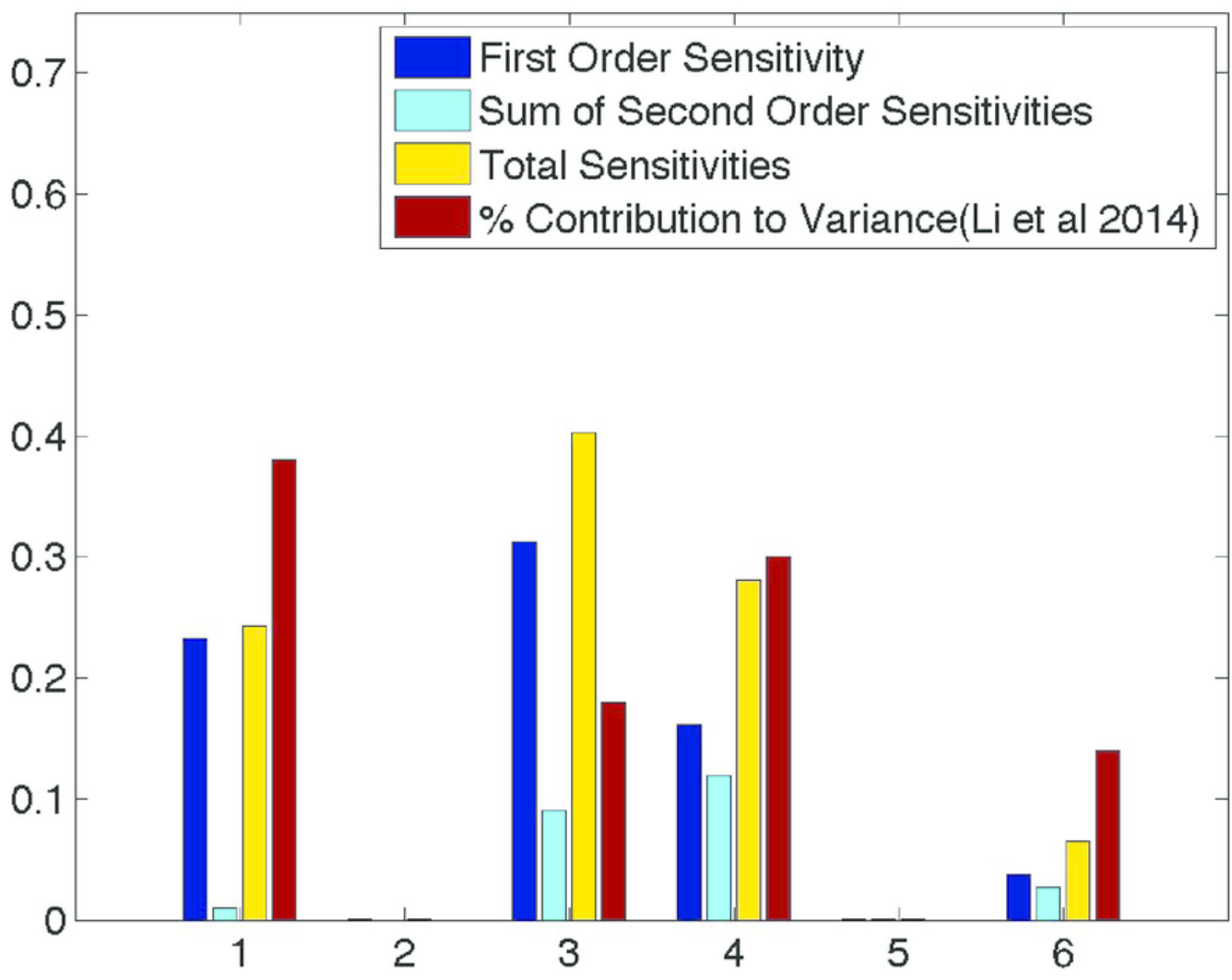

B.

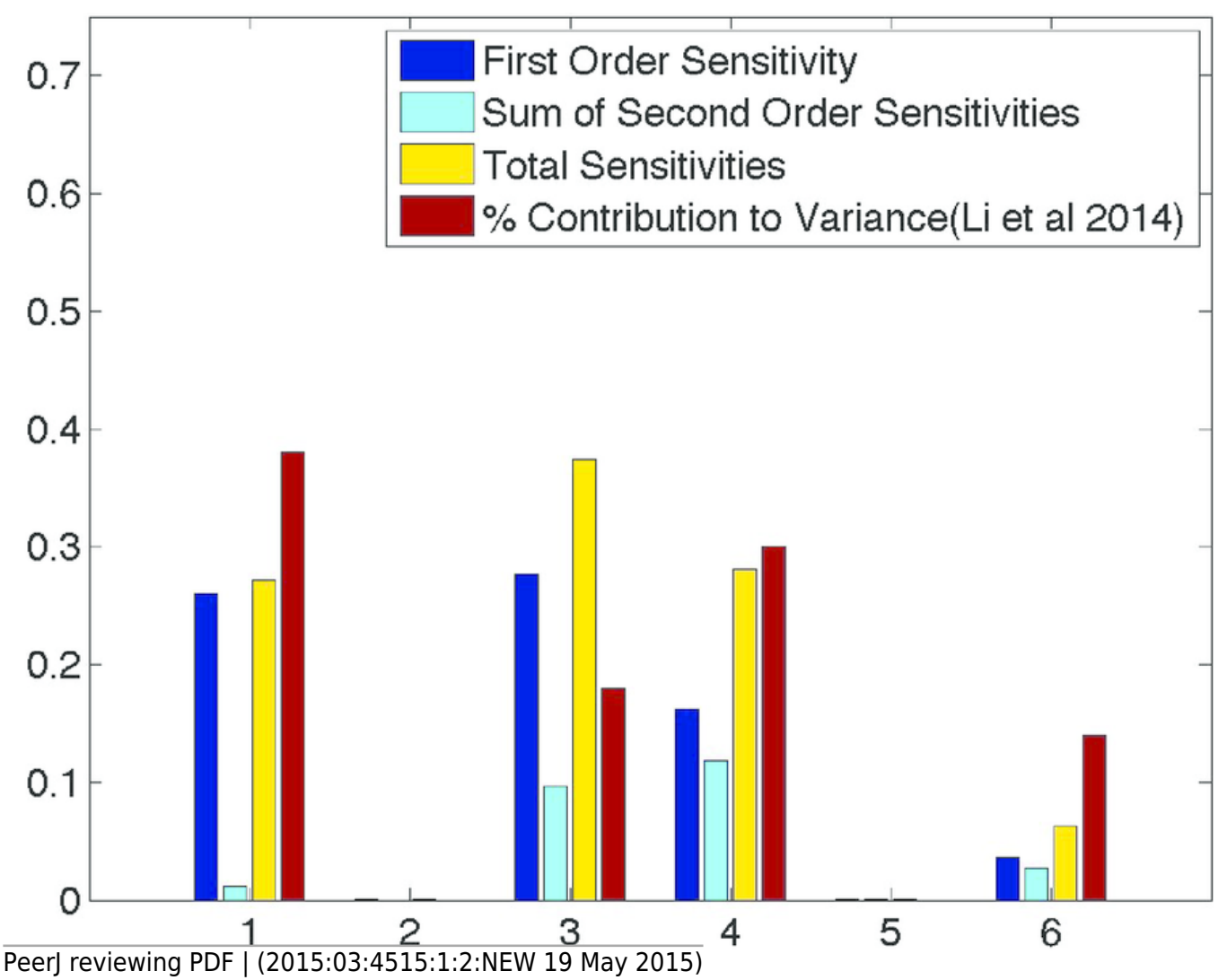


4

Temporal dynamics of parameter sensitivities.

A. First-order parameter sensitivities at the middle nucleus over time for a ubiquitous gene with initial concentrations of 1.0. B. First-order parameter sensitivities at the middle nucleus over time for an anterior maternally deposited gene. 
A.

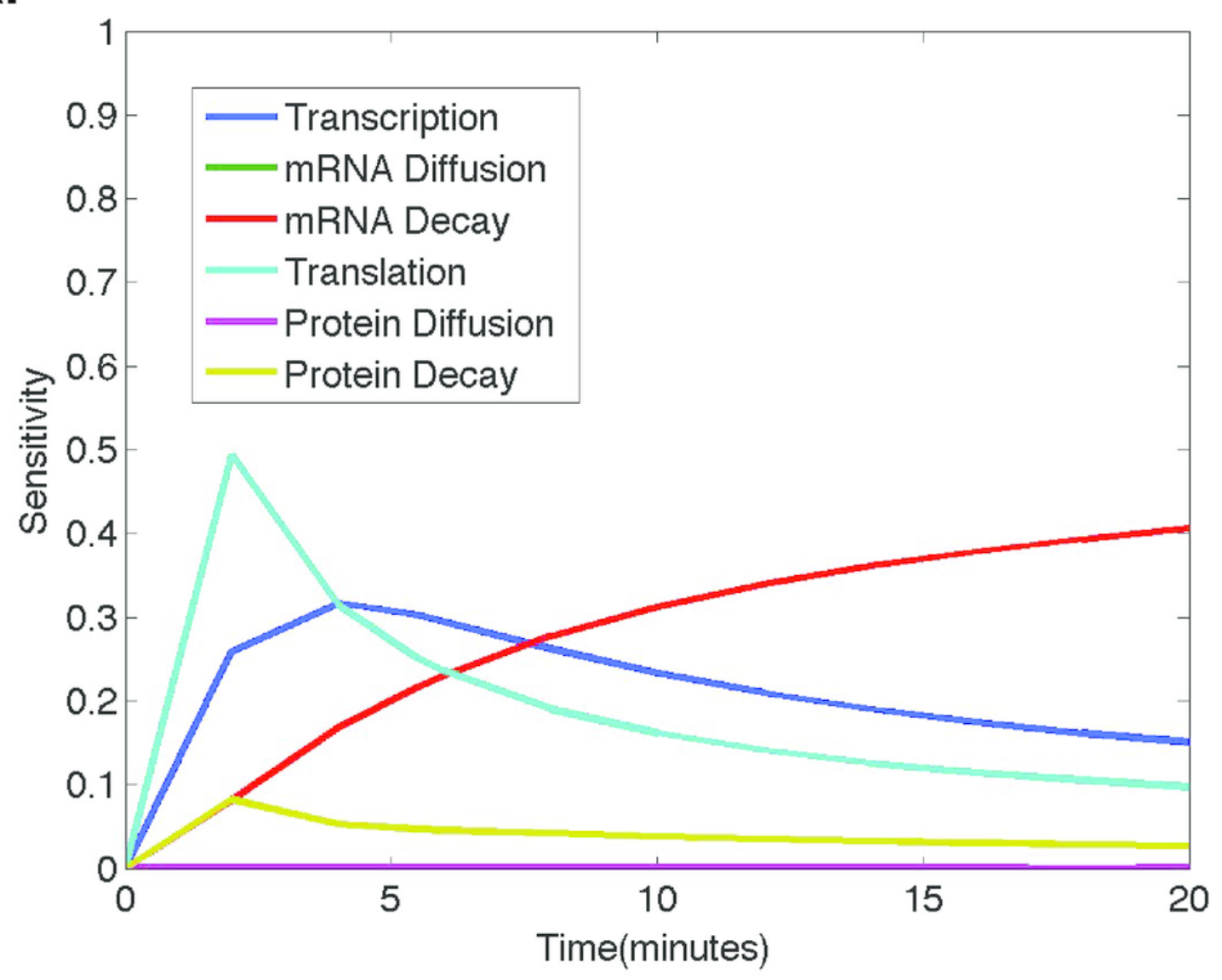

B.

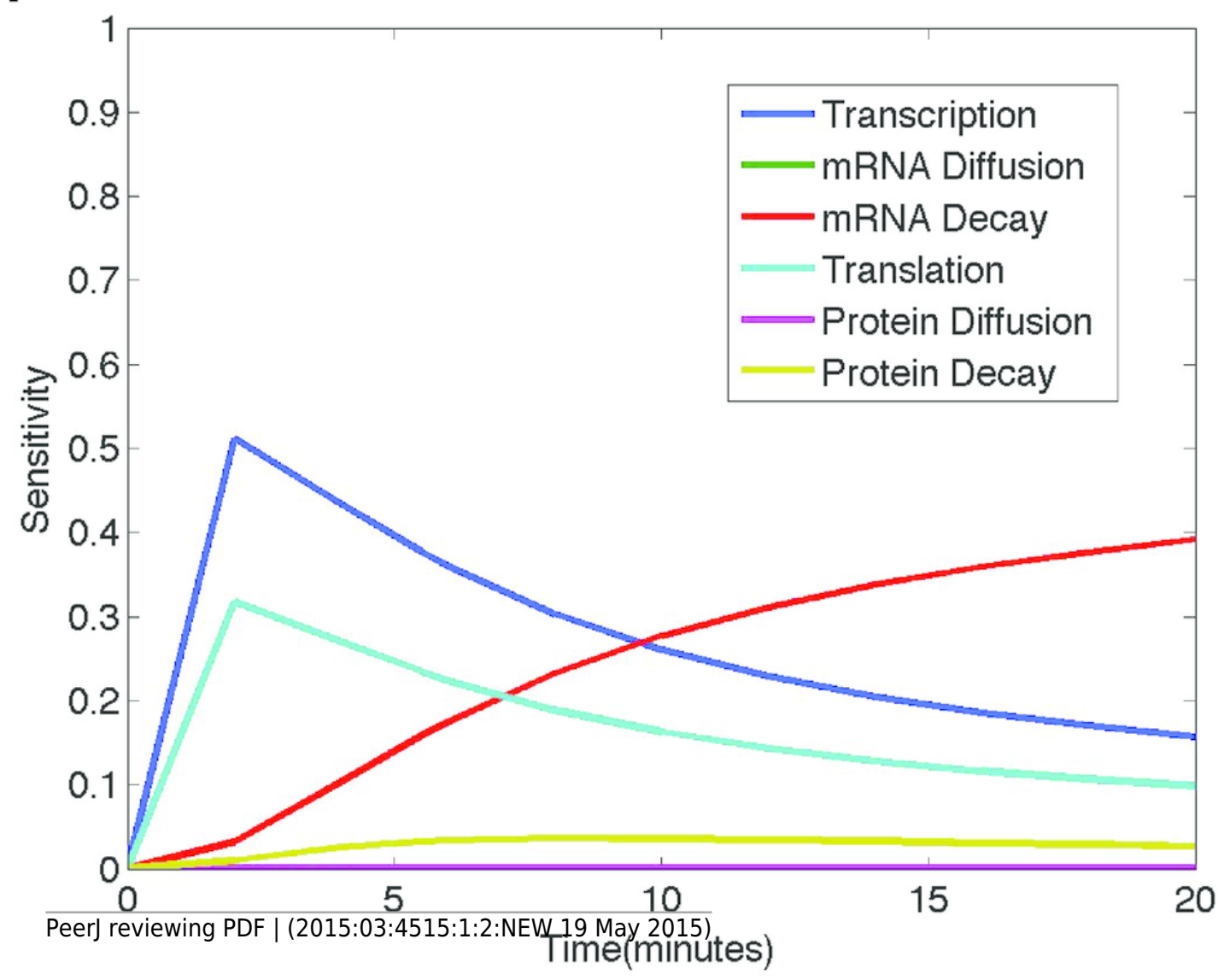

